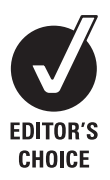

See Editorial, p 176

${ }^{1}$ Medical Research Institute of New Zealand, Wellington, New Zealand; ${ }^{2}$ Capital and Coast District Health Board, Wellington, New Zealand;

${ }^{3}$ University of Otago Wellington, Wellington, New Zealand;

${ }^{4}$ University of Southampton,

Southampton, UK

Correspondence to: Professor R Beasley, Medical Research Institute of New Zealand, PO Box 10055 , Wellington 6143, New Zealand; Richard.Beasley@mrinz.ac.nz

Accepted 1 July 2008 Published Online First 15 August 2008

\title{
Routine use of oxygen in the treatment of myocardial infarction: systematic review
}

\author{
M Wijesinghe, ${ }^{1} \mathrm{~K}$ Perrin, ${ }^{1}$ A Ranchord, ${ }^{2} \mathrm{M}$ Simmonds, ${ }^{2} \mathrm{M}$ Weatherall, ${ }^{3} \mathrm{R}$ Beasley ${ }^{1,4}$
}

\begin{abstract}
Context: International guidelines recommend the routine use of oxygen therapy in the treatment of myocardial infarction (MI).

Objective: To undertake a systematic review and metaanalysis of randomised placebo-controlled trials of oxygen therapy in Ml.

Data sources: Medline, Cochrane Database of Systematic Reviews, Cochrane Central Register of Controlled Trials, EMBASE and CINHAL.

Study selection: Randomised placebo-controlled trials of oxygen therapy in Ml.
\end{abstract}

Data extraction: The primary clinical outcome was mortality.

Results: Two of 51 potential studies met the inclusion criteria. The one study with substantive clinical outcome data reported that in uncomplicated Ml, high-flow oxygen was associated with a non-significant increased risk of death (risk ratio $2.9,95 \% \mathrm{Cl} 0.8$ to $10.3, p=0.08$ ) and a greater serum aspartate aminotransferase level (difference $19.2 \mathrm{IU} / \mathrm{ml}, 95 \% \mathrm{Cl} 0$ to 38.4, $\mathrm{p}=0.05$ ) than room air.

Conclusion: The limited evidence that does exist suggests that the routine use of high-flow oxygen in uncomplicated MI may result in a greater infarct size and possibly increase the risk of mortality.

Oxygen has been used in the treatment of myocardial infarction (MI) and acute coronary syndromes for over 100 years. ${ }^{1}$ The rationale for its longstanding use is that it increases oxygen delivery to the ischaemic myocardium, thereby reducing the size of the MI and improving clinical outcomes. Evidence in support of this approach is primarily derived from animal models, in which the administration of $100 \%$ oxygen under normobaric or hyperbaric conditions during and/or after experimental coronary artery occlusion reduces the extent of myocardial necrosis in some, ${ }^{2-6}$ but not all, studies..$^{7-10}$ However, concern has been expressed that the findings from animal studies may have poor generalisability to the clinical situation in humans. ${ }^{21}$ Furthermore, there is evidence that the routine use of high-flow oxygen in uncomplicated MI may not improve clinical outcomes and may potentially cause harm. ${ }^{11}{ }^{12}$ It is well established that arterial oxygen tension is a major determinant of coronary artery tone $e^{13-16}$ and that hyperoxia may result in a marked reduction in coronary artery blood flow. ${ }^{17-24}$ Other cardiovascular effects of high-flow oxygen therapy include a reduction in cardiac output and stroke volume and an increase in systemic vascular resistance and blood pressure in patients with a MI. ${ }^{25-31}$

Despite this conflicting evidence, contemporaneous international guidelines ${ }^{32}{ }^{33}$ recommend the routine use of supplemental oxygen in the treatment of MI. As the treatment of MI is at the forefront of evidence-based medicine, we sought to review the clinical efficacy and safety of the use of oxygen in this clinical situation. A systematic review was undertaken to identify randomised placebo-controlled trials of oxygen therapy in the treatment of acute MI with the intention of estimating differences in clinical outcomes by meta-analysis.

\section{METHODS}

\section{Search strategy}

To identify all studies that investigated the effect of oxygen therapy in MI, a search of studies containing the key words "oxygen" or "myocardial infarction" was conducted from Medline, Cochrane Database of Systematic Reviews, Cochrane Central Register of Controlled Trials, Embase and CINHAL to March 2007. The reference lists of all relevant studies were also examined. Particular attention was given to searching for studies before 1950, which would not be included in the electronic reference databases.

\section{Inclusion criteria}

Two people examined each paper's title and abstract and then the full paper if necessary. To be included in the review, studies had to be randomised, controlled, clinical trials of oxygen therapy in MI that reported clinical outcome measures.

\section{Exclusion criteria}

Any studies investigating the use of oxygen for any clinical indication other than MI, such as heart failure, were excluded. Non-randomised controlled trials, review articles, editorials and letters were also excluded.

Animal studies were excluded owing to the poor generalisability from laboratory animal models to patients because of differences in coronary anatomy, collaterals, haemodynamic responses and natural disease states such as coronary artery disease. $^{211}$ Studies using hyperbaric oxygen or intracoronary oxygen infusion were excluded as these therapeutic interventions are not commonly used in clinical practice and the physiological effects may differ from those of normobaric highflow oxygen therapy. As a result, the findings of such studies are not generalisable to consideration of the efficacy and safety of oxygen therapy in the treatment of MI.

\section{Data extraction}

Extraction of data was based on reported summary statistics (counts, means and standard deviations) 
for the intention-to-treat population. The primary outcome variable was in-hospital mortality. Secondary outcome variables were measures of infarct size by cardiac enzyme levels, occurrence of ventricular arrhythmia and opiate use. Where relevant, other clinical outcome variables reported in the clinical trials were noted. The trial quality was assessed using the standard Jadad score based on the adequacy of randomisation, blinding and follow-up, with a maximum score of five points.

\section{Data analysis}

As only two studies met the inclusion criteria and only one of these reported on the number of deaths in each treatment group formal meta-analysis was not undertaken. In the two identified studies, point estimates and confidence intervals were calculated for the main outcome variables, using relative risk for categorical variables and a $t$ test for continuous variables.

\section{RESULTS}

\section{Search results}

Figure 1 shows the QUOROM statement for the search, which identified 51 potentially relevant articles on the use of oxygen therapy in MI. From these, the 13 studies which investigated the use of hyperbaric or aqueous oxygen, six review articles, one letter, four editorials and two systematic reviews were excluded. An additional 23 studies were excluded, including case reports, case series, crossover studies, cohort studies and non-randomised trials of oxygen therapy in MI. Two studies met the criteria for inclusion in the systematic review s $^{35}$ and are presented in detail here.

\section{Study 1}

A study undertaken by Rawles and Kenmure. ${ }^{34}$

This parallel-group, double-blind controlled trial of oxygen therapy in uncomplicated MI randomised 200 patients with suspected MI to receive either oxygen or compressed air by

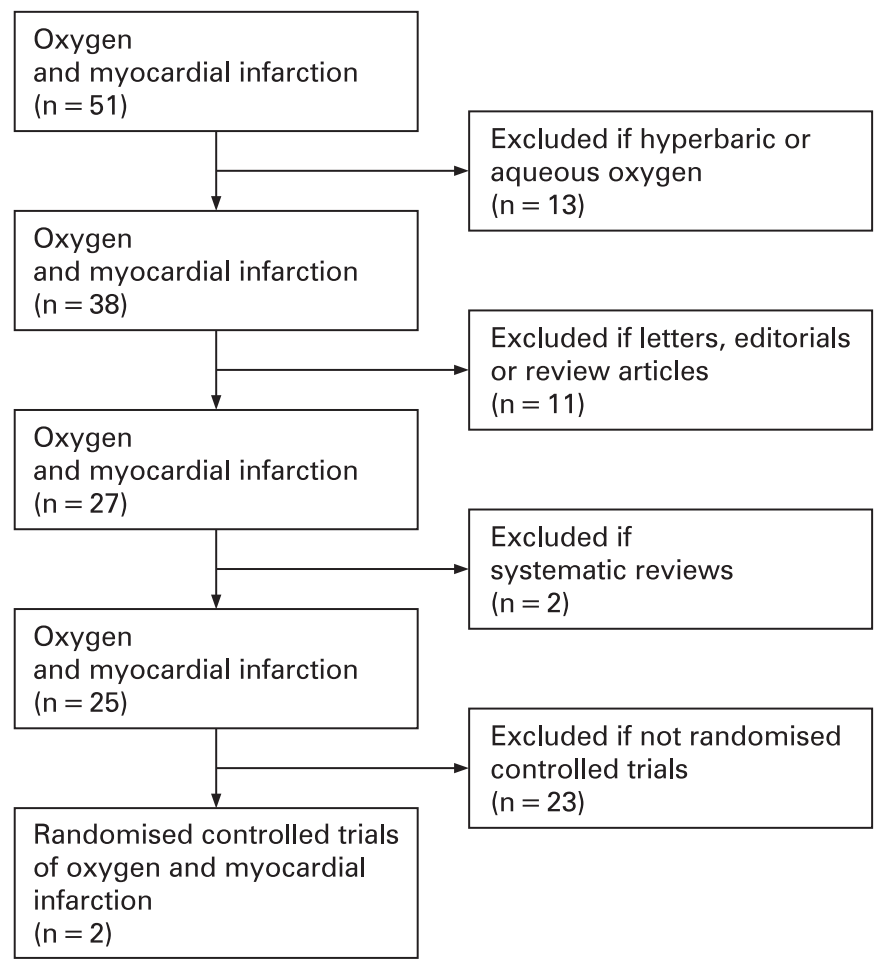

Figure 1 QUOROM statement for the search. means of a medium concentration mask at a flow rate of $6 \mathrm{l} / \mathrm{min}$ for $24 \mathrm{~h}$. Both patients and medical staff were blinded to the treatment received. Patients were excluded if they had clinical evidence of heart failure, chronic pulmonary disease or were breathless from any other cause, or if they had been transferred from other wards for the treatment of arrhythmias or had experienced a cardiac arrest before admission or had cardiogenic shock. Forty-three patients in whom MI was not subsequently confirmed were excluded from the analysis. The Jadad score for this study was $5 / 5$; however, there was no prespecified primary outcome variable or power calculation.

There were $9 / 80$ (11.3\%) deaths in the oxygen group and 3/77 $(3.9 \%)$ in the air group, relative risk of death 2.9 (95\% CI 0.8 to $10.3, p=0.08)$. The maximum serum aspartate aminotransferase level was used as a surrogate for infarct size, with mean (SD) values of 99.9 (63.1) and 80.7 (56.8) IU/ml in the oxygen and air groups respectively; difference $19.2 \mathrm{IU} / \mathrm{ml}$ (95\% CI 0 to 38.4 , $p=0.05)$. Ventricular tachycardia occurred in $11 / 80(13.8 \%)$ and $5 / 77(6.5 \%)$ of the oxygen and air groups, relative risk 2.1 (0.8 to $5.8, \mathrm{p}=0.13)$. Opiate use occurred in $57 / 80(71.3 \%)$ and $52 / 77$ $(67.5 \%)$ of the oxygen and air groups, relative risk 1.1 ( 0.9 to 1.3 , $\mathrm{p}=0.61)$.

The authors concluded that there was "suggestive evidence of a deleterious effect of oxygen" and that "there seems to be little place for routine oxygen administration to all patients with acute myocardial infarction".

\section{Study 2}

A study undertaken by Wilson and Channer. ${ }^{35}$

This parallel-group, non-blinded controlled study of oxygen therapy in MI was reported in conjunction with a postal survey on the use of pulse oximetry and supplemental oxygen in coronary care units in England. In the clinical study, 50 patients with MI who had received thrombolysis with streptokinase within $6 \mathrm{~h}$, were randomised to $4 \mathrm{l} / \mathrm{min}$ of oxygen or room air via face mask for $24 \mathrm{~h}$. However, results are only reported for 42 of the participants and one subject who died within the $24 \mathrm{~h}$ period of the study was excluded. The study was not blinded in regard to oxygen therapy. However, one of the aims of this study was to determine the ability of doctors to diagnose hypoxaemia clinically and, as a result, the oximetry recordings were blinded to both patient and doctor. Patients with central cyanosis, pulmonary disease requiring oxygen independent of cardiac status, those in whom blood gases showed a $\mathrm{PaCO}_{2}$ $>5.5 \mathrm{kPa}$ and patients with left ventricular failure requiring inotropic support were excluded from the trial. All patients were monitored with a pulse oximeter and Holter ECG monitoring continuously for the $24 \mathrm{~h}$ period of the study. The Jadad score was $5 / 5$; however, there was no prespecified primary outcome variable or power calculation. The purpose of the study was to document the occurrence of hypoxaemia and to measure the effect of supplemental oxygen on hypoxaemia rather than to compare outcomes measured by infarct size or mortality.

The authors did not state in which randomised group the death occurred and as a result, we were not able to calculate the risk of death for oxygen therapy. No surrogate measurement of infarct size was presented. Ventricular tachycardia occurred in $5 / 22(22.7 \%)$ and $5 / 20(25.0 \%)$ of the oxygen and air groups, relative risk 0.9 (0.3 to $2.7, \mathrm{p}=0.86)$. Opiate use occurred in $16 / 22(72.7 \%)$ and $18 / 20(90.0 \%)$ of the oxygen and air groups, relative risk 0.8 (0.6 to $1.1, \mathrm{p}=0.15)$.

Of interest $1 / 22(4.5 \%)$ participants who received oxygen had an episode of oxygen saturation below $80 \%$ compared with $7 / 20$ 
$(35.0 \%)$ of the participants who received air, relative risk 0.1 $(95 \%$ CI 0.02 to $1.0, p=0.01)$.

\section{DISCUSSION}

This systematic review and meta-analysis identified only two randomised placebo-controlled trials of high-flow oxygen therapy in the first $24 \mathrm{~h}$ after an uncomplicated MI, ${ }^{34}{ }^{35}$ with substantive clinical outcome data provided in only one study. ${ }^{28}$ Point estimates from the study with substantive reporting identified that high concentration oxygen resulted in a threefold increased mortality rate and greater infarct size, as measured by serum cardiac enzyme rise in patients with uncomplicated MI. Confidence intervals were wide and this study lacked statistical power to detect clinically important differences in other clinical outcomes. As a result we conclude that there is an absence of evidence to support the routine prescription of high-flow oxygen therapy in patients with uncomplicated MI. A pessimistic view of the available evidence is that the use of supplementary oxygen may cause harm when routinely used in this situation.

\section{Included studies}

As the evidence of potential harm from this systematic review is primarily derived from the Rawles and Kenmure study, ${ }^{34}$ it is necessary to consider its methodology and findings in some detail. The study was well designed with a Jadad score of 5, the high-flow oxygen and air regimens were administered double blind with the cylinders shrouded, and an intention-to-treat analysis was performed. Patients had uncomplicated MI and as a result, the findings do not related to MI complicated by heart failure, cardiogenic shock or arrhythmias. Furthermore, there does not appear to have been routine use of $\beta$ blockers, thrombolysis, percutaneous coronary intervention, or aspirin therapy in this trial and thus the findings may not be generalisable to current practice.

The $25 \%$ greater cardiac enzyme release was suggestive of a greater infarct size due to the high concentration oxygen therapy. The threefold increase in deaths in the oxygen treatment group can be considered from a number of perspectives. First, the study was not powered to determine a difference in mortality, with the primary aim to determine the effect of oxygen on the size of the infarction, incidence of arrhythmias and the use of analgesics. This is reflected in the wide confidence intervals consistent with either harm or benefit. Taking the upper limit, the number needed to cause a death might have been as small as 6.5 for oxygen therapy. These findings provide no support for the view that oxygen therapy was either beneficial or safe, which would be necessary to justify its use as a routine therapeutic intervention. In contrast, this study suggests that the routine use of high-flow oxygen in uncomplicated MI may result in a greater infarct size and, possibly, increase the risk of mortality.

The other study included in the systematic review ${ }^{35}$ did not report, and in any case lacked statistical power to investigate, our nominated primary outcome of death. The secondary outcomes of ventricular tachycardia occurrence and use of opiates were not different between the two groups, despite a higher prevalence of severe hypoxaemia in the group receiving air. These findings are consistent with previous studies that reported no relationship between hypoxia and ischaemia in coronary artery disease, ${ }^{36}{ }^{37}$ but contrasted with another study that demonstrated a temporal relationship between nocturnal hypoxaemia and both ST-segment depression and arrhythmia after a MI. ${ }^{38}$

\section{Potential mechanisms}

This interpretation of potential harm with high concentration oxygen therapy is consistent with its known haemodynamic effects. In patients with MI, hyperoxia reduces cardiac output and stroke volume and increases the mean arterial pressure and systemic vascular resistance. ${ }^{25-31}$ If the baseline arterial oxygen saturations are $>90 \%$, high concentration oxygen does not increase oxygen transport, as the reductions in cardiac output are in excess of the increase in oxygen content. ${ }^{26}$ The adverse haemodynamic responses are greatest in patients with MI not complicated by heart failure or cardiogenic shock. ${ }^{39}$

More importantly, there is also substantive evidence that arterial oxygen tension is a major determinant of coronary artery regulatory tone, ${ }^{13-16}$ and that high concentration oxygen therapy resulting in hyperoxia reduces coronary artery blood flow, ${ }^{17-24}$ although an increase in collateral blood flow to the ischaemic myocardium has been reported in an animal model. ${ }^{40}$ The magnitude of the reduction in coronary blood flow with hyperoxia may be substantial in patients with coronary artery disease. This has been illustrated by two studies ${ }^{19}{ }^{20}$ which used the measurement of intracoronary Doppler flow in subjects with stable coronary artery disease. Breathing 100\% oxygen by face mask for 10-15 min decreased coronary blood flow by 20 $30 \%$ in association with a $23-40 \%$ increase in coronary resistance. Oxygen breathing elicited these changes without affecting the diameter of the large conduit coronary arteries. These findings indicate that hyperoxia is a potent vasoconstrictor stimulus to the coronary circulation, functioning at the level of the microvascular resistance vessels. These effects are likely to be secondary to an effect on coronary endothelial function, with the accelerated oxidative degradation of coronary endothelium-derived nitric oxide by reactive oxygen species. ${ }^{19} 20$

Another potential mechanism is that hyperoxia resulting from high concentration oxygen therapy may exacerbate reperfusion injury to the heart owing to the increased production of oxygen-free radicals. ${ }^{41}$ This may be particularly relevant to the current therapeutic goal in patients with STsegment elevation myocardial infarction of achieving urgent reperfusion of the ischaemic myocardium by restoration of coronary blood flow by thrombolysis or percutaneous coronary intervention. ${ }^{42}$

\section{Methodological concerns}

The main methodological concern is whether all available studies were included in the systematic review. We consider that it is likely that we have identified all eligible randomised controlled trials because of the comprehensive nature of the search, including non-English language publications and the extensive review of published manuscripts, systematic reviews and guidelines. Although electronic databases do not reference studies before 1950 we are confident that our search strategy identified almost all potential studies during this period owing to our comprehensive review of published papers.

Four studies were identified in the systematic review that indicated benefit with the use of oxygen in angina or MI, but did not meet our inclusion criteria. Three studies reported benefit with oxygen therapy in pacing-induced angina ${ }^{43}$ and exercise-induced angina, ${ }^{44}{ }^{45}$ although similar benefits could not be demonstrated in later studies. ${ }^{46}{ }^{47}$ The other study was that of Madias et $a l,{ }^{48}$ who suggested that the administration of 
oxygen therapy to patients experiencing a MI may reduce ischaemic injury, based on precordial ST-segment mapping. Unfortunately, the lack of a randomised control design, standardisation of the duration of oxygen therapy, or blinding in the ST measurements, limited the significance of these findings.

The major evidence for the benefit of oxygen therapy in MI comes from studies of experimental coronary occlusion in animal models. Some $e^{2-6}$ but not all ${ }^{7-10}$ of these studies have reported that high concentration oxygen therapy under normobaric or hyperbaric conditions reduces the size of the MI. However, the extrapolation of these findings to the clinical setting is uncertain, particularly because of differences in coronary anatomy and collaterals, which are much more extensive in animal models such as the dog. ${ }^{21}$ Furthermore, experimental animals had normal coronary vessels and myocardial function, whereas most patients with MI have diffuse coronary obstructive lesions and myocardial damage. Likewise, the findings from animal studies of hyperbaric oxygen are not generalisable to the use of oxygen therapy under normobaric conditions in MI. This restriction also applies to human studies of hyperbaric oxygen, ${ }^{49}$ or intracoronary infusion of aqueous oxygen $^{50}$ in MI.

\section{Guidelines}

Major international guidelines have taken the pragmatic approach of recommending routine oxygen therapy in the treatment of uncomplicated $\mathrm{MI}^{32}{ }^{33}$ which is consistent with widespread clinical practice. If the American Heart Association is taken as an example, it states that "it is reasonable to administer supplementary oxygen to all patients with uncomplicated ST-segment elevation myocardial infarction during the first 6 hours" as level C evidence. However, it is acknowledged in the accompanying text that it is "not known whether this therapy limits myocardial damage or reduces morbidity or mortality". The two studies referenced in support of oxygen therapy are the animal study of experimental coronary artery occlusion $^{2}$ and the human precordial ECG mapping study ${ }^{48}$ discussed above. We respectfully suggest that these recommendations are not supported by the balance of evidence and at best can be considered level I (insufficient evidence) or at worst level $\mathrm{C}$ evidence of harm. The recommendations of the New Zealand Branch of the Cardiac Society of Australia and New Zealand may be preferred, ${ }^{42}$ in which it is recommended that "oxygen should be administered to keep the saturations around 96\%" as level D evidence. Hypoxaemia may commonly occur in $\mathrm{MI}^{35} 3951$ and oxygen therapy may be indicated to relieve hypoxaemia identified by oximetry monitoring in this situation.

\section{CONCLUSIONS}

We conclude by suggesting that there is insufficient evidence to support the routine use of high-flow oxygen in the treatment of uncomplicated MI. The balance of the limited evidence that exists suggests that the routine use of oxygen in this situation may increase infarct size and possibly increase the risk of mortality, owing to its haemodynamic effects, including a reduction in coronary blood flow. Major international guidelines do not appear to represent the current evidence base and may need revision. There is an urgent requirement for randomised controlled trials of the use of oxygen therapy in MI that are sufficiently powered to enable the risk of mortality to be assessed.

\section{Competing interests: None.}

MWi is a Wellington Hospitals and Health Foundation Research Fellow and KP is a Health Research Council of New Zealand Training Fellow. MWi and KP undertook the systematic review, MWe undertook the statistical analyses and all authors reviewed the studies identified and contributed to writing the manuscript.

\section{REFERENCES}

1. Steele C. Severe angina pectoris relieved by oxygen inhalations. BMJ 1900;2:1568

2. Maroko PR, Radvany P, Braunwald E, et al. Reduction of infarct size by oxygen inhalation following acute coronary occlusion. Circulation 1975:52:360-8.

3. Kelly RF, Hursey TL, Parrillo JE, et al. Effect of $100 \%$ oxygen administration on infarct size and left ventricular function in a canine model of myocardial infarction and reperfusion. Am Heart J 1995:130:957-65.

4. Whalen RE, Saltzman HA. Hyperbaric oxygenation in the treatment of acute myocardial infarction. Prog Cardiovasc Dis 1968;10:575-83.

5. Kawamura M, Sakakibara K, Sakakibara B, et al. Protective effect of hyperbaric oxygen for the temporary ischaemic myocardium: macroscopic and histological data. Cardiovasc Res 1976:10:599-604.

6. Thomas MP, Brown LA, Sponseller DR, et al. Myocardial infarct size reduction by the synergistic effect of hyperbaric oxygen and recombinant tissue plasminogen activator. Am Heart J 1990;120:791-800.

7. Mogelson S, Davidson J, Sobel BE, et al. The effect of hyperbaric oxygen on infarct size in the conscious animal. Eur J Cardiol 1980;12:135-46.

8. Robertson HF. The effect of hyperbaric oxygenation on myocardial infarction in dogs. Can J Surg 1966;9:81-90.

9. Malm A, Arborelius M Jr, Bornmyr S, et al. Effects of oxygen on acute myocardial infarction: a thermographic study in the dog. Cardiovasc Res 1977;11:512-8.

10. Shnier CB, Cason BA, Horton AF, et al. Hyperoxemic reperfusion does not increase myocardial infarct size. Am J Physiol 1991;260(Pt 2):H1307-12.

11. Nicholson C. A systematic review of the effectiveness of oxygen in reducing acute myocardial ischaemia. J Clin Nurs 2004;13:996-1007.

12. Beasley R, Aldington $S$, Weatherall $M$, et al. Oxygen therapy in myocardia infarction: an historical perspective. J $R$ Soc Med 2007:100:130-3.

13. Hilton R, Eichholtz $F$. The influence of chemical factors on the coronary circulation. J Physiol 1925;59:413-25

14. Markwalder J, Starling EH. A note on some factors which determine the blood-flow through the coronary circulation. J Physiol 1913;47:275-85.

15. Gellai M, Norton JM, Detar R. Evidence for direct control of coronary vascular tone by oxygen. Circ Res 1973;32:279-89.

16. Berne RM, Blackmon JR, Gardner TH. Hypoxemia and coronary blood flow. J Clin Invest 1957:36:1101-6.

17. Sobol BJ, Wanlass SA, Joseph EB, et al. Alteration of coronary blood flow in the dog by inhalation of 100 per cent oxygen. Circ Res 1962;11:797-802.

18. Baron JF, Vicaut $\mathrm{E}, \mathrm{Hou} \mathrm{X}$, et al. Independent role of arterial $\mathrm{O}_{2}$ tension in local control of coronary blood flow. Am J Physiol 1990;258(Pt 2):H1388-94.

19. McNulty PH, King N, Scott S, et al. Effects of supplemental oxygen administration on coronary blood flow in patients undergoing cardiac catheterization. Am J Physiol Heart Circ Physiol 2005;288:H1057-62.

20. McNulty PH, Robertson BJ, Tulli MA, et al. Effect of hyperoxia and vitamin $\mathrm{C}$ on coronary blood flow in patients with ischemic heart disease. J Appl Physiol 2007:102:2040-5

21. Ratliff NB, Hockel DB, Mikat E. Myocardial oxygen metabolism and myocardial blood flow in dogs in hemorrhagic shock: effects of hyperbaric oxygen. Circ Res 1969;24:901-9.

22. Ishikawa K, Lee T, Ganz W. Effect of oxygen on perfusion and metabolism of the ischemic myocardium. J Appl Physiol 1974;36:56-9.

23. Kenmure ACF, Beatson JM, Cameron AJV, et al. Effects of oxygen on myocardial blood flow and metabolism. Cardiovasc Res 1971;5:483-9.

24. West JW, Guzman SV. Coronary dilatation and constriction visualized by selective arteriography. Circ Res 1959;7:527-36.

25. Loeb HS, Chuquimia R, Sinno MZ, et al. Effects of low-flow oxygen on the hemodynamics and left ventricular function in patients with uncomplicated acute myocardial infarction. Chest 1971;60:352-5.

26. Sukumalchantra $\mathbf{Y}$, Levy S, Danzig R, et al. Correcting arterial hypoxemia by oxygen therapy in patients with acute myocardial infarction. Effect on ventilation and hemodynamics. Am J Cardiol 1969;24:838-52.

27. Kenmure AC, Murdoch WR, Beattie AD, et al. Circulatory and metabolic effects of oxygen in myocardial infarction. BMJ 1968;4:360-4.

28. Thomas $\mathbf{M}$, Rea M. Haemodynamic effects of oxygen in patients with acute myocardial infarction. Heart 1965;27:401-7.

29. Shillingford JP, Thomas M. Cardiovascular and pulmonary changes in patients with myocardial infarction treated in an intensive care and research unit. Am J Cardiol 1967;20:484-93.

30. Foster GL, Casten CG, Reeves TJ, et al. The effects of oxygen breathing in patients with acute myocardial infarction. Cardiovasc Res 1969;3:179-89.

31. Mackenzie GJ, Flenley DC, Taylor SH, et al. Circulatory and respiratory studies in myocardial infarction and cardiogenic shock. Lancet 1964;2:825-32.

32. Antman EM, Anbe DT, Armstrong PW, et al. ACC/AHA Guidelines for the Management of Patients with ST-Elevation Myocardial Infarction-executive summary. J Am Coll Cardiol 2004:44:671-719. 
33. Van de Werf F, Ardissino D, Betriu A, et al. Task Force on the Management of Acute Myocardial Infarction of the European Society of Cardiology. Management of acute myocardial infarction in patients presenting with ST-segment elevation. Eur Heart J 2003;24:28-66.

34. Rawles JM, Kenmure AC. Controlled trial of oxygen in uncomplicated myocardial infarction. BMJ 1976;1:1121-3.

35. Wilson AT, Channer KS. Hypoxaemia and supplemental oxygen therapy in the first 24 hours after myocardial infarction: the role of pulse oximetry. $J R$ Coll Physicians Lond 1997;31:657-61.

36. Smith HL, Sapsford DJ, Delaney ME, et al. The effect on the heart of hypoxaemia in patients with severe coronary artery disease. Anaesthesia 1996;51:211-8.

37. Entwhistle MD, Sommerville D, Tandon AP, et al. Effect of hypoxaemia on the resting electrocardiogram (ECG) in patients with cardiac ischaemia. Ann Acad Med Singapore 1994;23:460-4.

38. Galatius-Jensen S, Hansen J, Rasmussen V, et al. Nocturnal hypoxaemia after myocardial infarction: association with nocturnal myocardial ischaemia and arrhythmias. Heart 1994;72:23-30.

39. Davidson RM, Ramo BW, Wallace AG, et al. Blood-gas and hemodynamic responses to oxygen in acute myocardial infarction. Circulation 1973;47:704-11

40. Ribeiro LGT, Louie EK, Davis MA, et al. Augmentation of collateral blood flow to the ischaemic myocardium by oxygen inhalation following experimental coronary artery occlusion. Cardiovasc Res 1979;13:160-6.

41. Kaneda T, Ku K, Inoue T, et al. Postischemic reperfusion injury can be attenuated by oxygen tension control. Jpn Circ J 2001;65:213-8.
42. Non-ST-Elevation Myocardial Infarction Guidelines Group and the New Zealand Branch of the Cardiac Society of Australia and New Zealand. STelevation myocardial infarction: New Zealand management guidelines. N Z Med J 2005:118:U1680.

43. Horvat M, Yoshida S, Prakash R, et al. Effect of oxygen breathing on pacing-induced angina pectoris and other manifestations of coronary insufficiency. Circulation 1972; 45:837-44.

44. Riseman JEF, Brown MG. The effect of oxygen on the exercise tolerance of patients with angina pectoris. Am Heart J 1939;18:150-2.

45. Barach $\mathbf{A L}$, Pons ER, Berg J. Early use of oxygen in coronary thrombosis. JAMA 1960;74:130-5

46. Russek HI, Regan FD, Naegele CF. One hundred percent oxygen in the treatment of acute myocardial infarction and severe angina pectoris. JAMA 1950;144:373-5.

47. Lecerof H. Central haemodynamics during oxygen breathing in angina pectoris. Thorax 1974:29:673-5.

48. Madias JE, Madias NE, Hood WB Jr. Precordial ST-segment mapping. 2. Effects of oxygen inhalation on ischemic injury in patients with acute myocardial infarction. Circulation 1976:53:411-7.

49. Stavitsky Y, Shandling AH, Ellestad MH, et al. Hyperbaric oxygen and thrombolysis in myocardial infarction: the 'HOT MI' randomized multicenter study. Cardiology 1998:90:131-6.

50. Warda HM, Bax JJ, Bosch JG, et al. Effect of intracoronary aqueous oxygen on left ventricular remodeling after anterior wall ST-elevation acute myocardial infarction. Am J Cardiol 2005;96:22-4

51. Lal S, Savidge RS. Oxygen administration after myocardial infarction. Lancet 1969;1:890.

\section{BMJ Careers online re-launches}

BMJ Careers online has re-launched to give you an even better online experience. You'll still find our online services such as jobs, courses and careers advice, but now they're even easier to navigate and quicker to find.

New features include:

- Job alerts - you tell us how often you want to hear from us with either daily or weekly alerts

- Refined keyword searching making it easier to find exactly what you want

- Contextual display - when you search for articles or courses we'll automatically display job adverts relevant to your search

- Recruiter logos linked directly to their organisation homepage - find out more about the company before you apply

- RSS feeds now even easier to set up

Visit careers.bmj.com to find out more. 